SPICA Workshop, 03007 (2009)

DOI:10.1051/spica/200903007

(C) Owned by the authors, published by EDP Sciences, 2009

This is an Open Access article distributed under the terms of the Creative Commons Attribution-Noncommercial License, which permits unrestricted use, distribution, and reproduction in any noncommercial medium, provided the original work is properly cited.

\title{
EVOLVING GAS AND DUST IN THE GALAXY AND GALAXIES TO BE SEEN BY SPICA
}

\author{
Takashi Onaka $^{1}$, Itsuki Sakon ${ }^{1}$, Hidehiro Kaneda ${ }^{2}$, and Hideyuki Izumura ${ }^{3}$ \\ ${ }^{1}$ Department of Astronomy, Graduate School of Science, The University of Tokyo, Bunky-ku, Tokyo 113-0033, Japan \\ ${ }^{2}$ Gradunate School of Science, Nagoya University, Chikusa-ku, Nagoya 464-8602, Japan \\ ${ }^{3}$ Okayama Astronomical Observatory, National Astronomical Observatory of Japan, Asakuchi, Okayama, 719-0232, Japan
}

\begin{abstract}
Supernovae (SNe) and low-mass evolved stars eject elements synthesized in stellar interior to the interstellar space as solid particles, whereas dust grains are destroyed efficiently in SN shocks. A large fraction of interstellar dust must in fact be formed in dense clouds rather than being supplied by stellar sources. All of these processes in our Galaxy and nearby galaxies can be studied most effectively by imaging spectroscopy in the mid- to far-infrared instruments on board SPICA, only which allow us to investigate in detail the nature of dust grains as well as the abundance and physical conditions of gas species in the various regions in galaxies. This report summarizes the discussions of the science working group in Japan with the emphasis on investigations of the dust formation in stellar sources and dense clouds, and the material evolution in nearby galaxies that only SPICA can carry out.
\end{abstract}

Key words: dust: formation - dust: destruction - supernovae - supernova remnants - AGB stars - galaxies infrared observations - missions: SPICA

\section{INTRODUCTION}

Matter evolves as it switches their whereabouts from solid to gas or gas to solid in the universe and the dust life cycle in the interstellar medium (ISM) plays a critical role in the evolution of the universe. The cycling and evolution of dust involve mass-loss or dust-ejection process from evolved stars, dust processing in the interstellar space, and star-formation process in dense regions. The dust supply rate from evolved low-mass (Asymptotic Giant Branch: AGB) stars is relatively well studied and thought to be a major stellar source for interstellar dust grain. Dust supply from supernovae ( $\mathrm{SNe}$ ) is estimated to be larger, if all the condensible material becomes solid particles. However, the fraction and composition of the dust formed in $\mathrm{SNe}$ presently remain uncertain (Tielens, 2005). On the other hand, dust grains are destroyed very efficiently in the ISM by SN shocks (e.g. Jones et al., 1996). A naive consequence of this mass balance of dust grains suggests that most ISM dust should be formed in the interstellar space, i.e., dense clouds (Seab, 1988; Onaka, 2000). However very little is known for dust formation in the ISM.
All of these processes relating to the transmigration of dust grains can be studied most efficiently in the infrared, in particular with the mid- to far-infrared (MIR to FIR) spectroscopy. SPICA will make a crucial contribution to the understanding of the dust life cycle in the ISM owing to its unprecedented sensitivity and spatial resolution in this spectral range (Nakagawa \& Murakami, 2007; Swinyard et al., 2009). In the following the discussion of the ISM science working group in Japan is summarized, concentrating on six particular topics rather than reviewing all possible items in the shopping list.

\section{DUST FORMATION IN MASSIVE STARS}

$\mathrm{SNe}$ are the major supplier of heavy elements to the ISM and supposed to be a main dust supplier if all the condensible elements are taken into solid particles (Tielens, 2005). Recent detection of dust in high redshift objects suggests a high efficiency of dust formation in $\mathrm{SNe}\left(>0.1 \mathrm{M}_{\odot}\right.$ per $\mathrm{SN}$ ), which is also supported by theoretical predictions (e.g. Todini \& Ferrara, 2001; Nozawa et al., 2003). Latest observations of SNe with Spitzer and AKARI, however, all indicate that the amount of dust grains formed in SN ejecta is much smaller than the expectation (e.g. Sakon et al., 2009; Kotak et al., 2009).

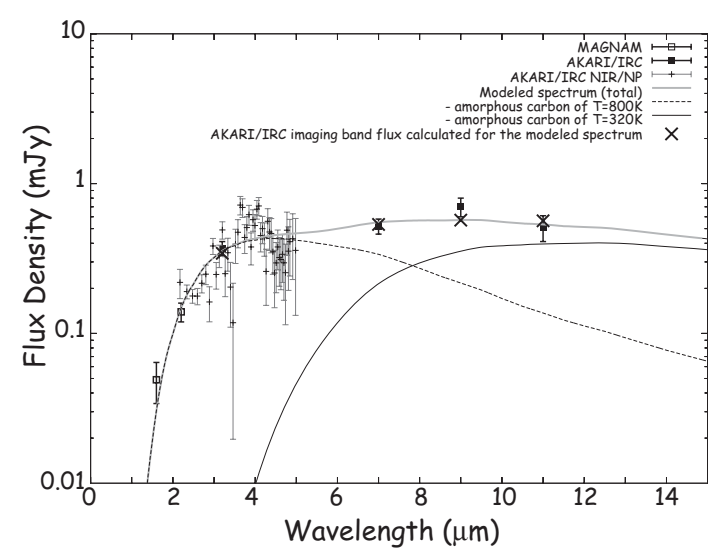

Figure 1. AKARI /IRC (filled squares) and MAGNUM (open squares) data of SN2006jc taken at 200 days after the explosion. The near-infrared spectrum taken with the AKARI/IRC is also plotted. The gray line indicates the best-fit model of two components of amorphous carbon dust (Sakon et al., 2009). 

Figure 1 shows $A K A R I$ observations of SN 2006jc (Sakon
et al., 2009), indicating the multi-component nature of the IR emission. Sakon et al. (2009) attribute it to the emission from dust formed in the ejecta (hot component) and that from circumstellar dust formed prior to the SN explosion (warm component), deriving the dust mass in the ejecta to be $6.9 \pm 0.5 \times 10^{-5} \mathrm{M}_{\odot}$. This result indicates that the dust formed in the pre-SN phase may have a comparable contribution to the dust supply to that of SNe and should be investigated in detail. It should be noted, however, that because of the presence of the warm component and the lack of observations longer than $10 \mu \mathrm{m}$, there is still a possibility of undetected "warm" dust in the ejecta and "cold" dust in the circumstellar region. These observations of $\mathrm{SNe}$ indicate the importance of a wide spectral coverage in the MIR to FIR to correctly estimate the dust mass. Also it should be stressed that high spatial resolution is crucial to separate the contribution from the mother galaxy.

$\mathrm{SiO}$ molecule fundamental emission in 7.7-9.5 $\mu \mathrm{m}$ has been detected in SN 1987A (Roche et al., 1991; Wooden et al., 1993) and more recently in SN 2005af (Kotak et al., 2006). Kotak et al. (2009) reported the detection of the silicate emission for the first time in addition to the $\mathrm{SiO}$ fundamental in SN 2004et. The presence of $\mathrm{SiO}$ molecule is directly linked to the silicate dust formation and high spectral resolution observations of the $\mathrm{SiO}$ fundamental emission with SPICA will be very interesting to understand the silicate formation process in $\mathrm{SNe}$ as well as $\mathrm{CO}$ fundamental at $4.5-4.7 \mu \mathrm{m}$ (see next section).

We propose to make systematic monitoring observations of several $(>5)$ SNe in nearby galaxies $(<25 \mathrm{Mpc})$ with MIR to FIR imaging spectroscopy of SPICA to study dust formation process and dust properties (mass and composition) in SN ejecta. Based on the brightness of $\mathrm{SNe}$ so far observed, we should be able to observe these nearby $\mathrm{SNe}$ within 1-2 years after the explosion. High spectral resolution spectroscopy in $4-10 \mu \mathrm{m}$ of $\mathrm{CO}$ and $\mathrm{SiO}$ fundamentals will also provide significant information on the dust formation process.

\section{OBSERVATIONS OF SUPERNOVA REMNANTS}

While monitoring observations of SNe will provide us with a great deal of information on the dust formation process, the occurrence of SNe cannot be predicted and their location may not be suitable to the SPICA visibility for longterm monitoring. Supernova remnants (SNRs) provide an alternative stable target to study dust grains associated with SN explosion as well as dust destruction process in SN shocks. IR emission from SNRs in our Galaxy suffers severe confusion and high spatial resolution in FIR is indispensable to extract the IR emission from SNRs.

IR emission associated with SN ejecta has been reported only for a handful of SNRs (e.g. Ghavamian et al., 2009 , references therein). The total mass of the dust asso- ciated with SNRs is also much less than $0.1 \mathrm{M}_{\odot}($ e.g. Rho et al., 2008). It is also not an easy task to separate the contribution of the emission of dust in the SN ejecta from that of dust in the circumstellar region. Figure 2 shows AKARI images of SNR G292.0+1.8 (Lee et al., 2009). The dust emission from the SN ejecta is clearly seen in the 15 to $24 \mu \mathrm{m}$ band ratio image (right figure), suggesting the presence of a characteristic dust feature in this spectral range and the significance of multi-band observations. In fact the IRS spectroscopy indicates the presence of a broad feature around $18 \mu \mathrm{m}$ at the ejecta position of G292.0+1.8 (Ghavamian et al., 2009). A similar broad feature in 18$24 \mu \mathrm{m}$ has also been reported for other SNRs (Tappe et al., 2006; Rho et al., 2008, 2009b).
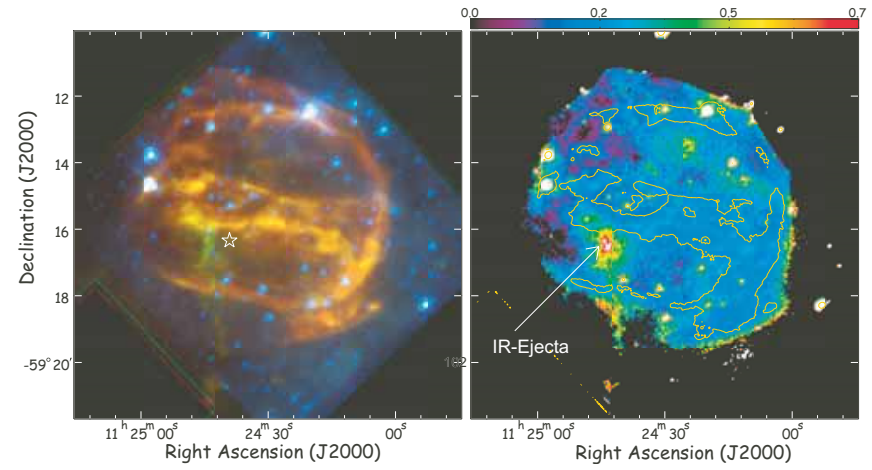

Figure 2. (Left) AKARI /IRC three-color infrared images ( $7 \mu \mathrm{m}$ in blue, $15 \mu \mathrm{m}$ in green and $24 \mu \mathrm{m}$ in red) of the SNR G292.0+1.8 (Lee et al., 2009). The star symbol indicates the location of the pulsar J1124-5916. (Right) The background subtracted 15 to $24 \mu \mathrm{m}$ band ratio image, where the ejecta position is indicated by the arrow.

A broad feature around $22 \mu \mathrm{m}$ has been reported in the Carina nebula and other massive star-forming regions, suggesting a possible connection of the broad feature to dust grains formed in SNe (Chan \& Onaka, 2000; Onaka et al., 2009). Figure 3 shows a comparison of the spectrum of the Carina nebula with those of Cas A and Sharpless 171 star-forming region (Onaka et al., 2009). While the Cas A feature peaks at a shorter wavelength and the profile is not exactly the same, they could be accounted for by the same dust species with the different shape distribution, which may result from dust processing.

The first overtone $\mathrm{CO}$ emission around $2.3 \mu \mathrm{m}$ has been detected in several SNe, while Rho et al. (2009a) report its first likely detection in Cas A. Detection of CO molecule in SNe and SNRs is significant since theoretical investigations suggest a possibility of direct condensation of carbon dust without forming $\mathrm{CO}$ in a rapidly cooling gas of SN ejecta (Clayton et al., 1999, 2001). If CO molecule is formed, then the production efficiency of carbon dust will be largely reduced. High spectral resolution observations of CO fundamental around $4.5-4.7 \mu \mathrm{m}$ with SPICA are 


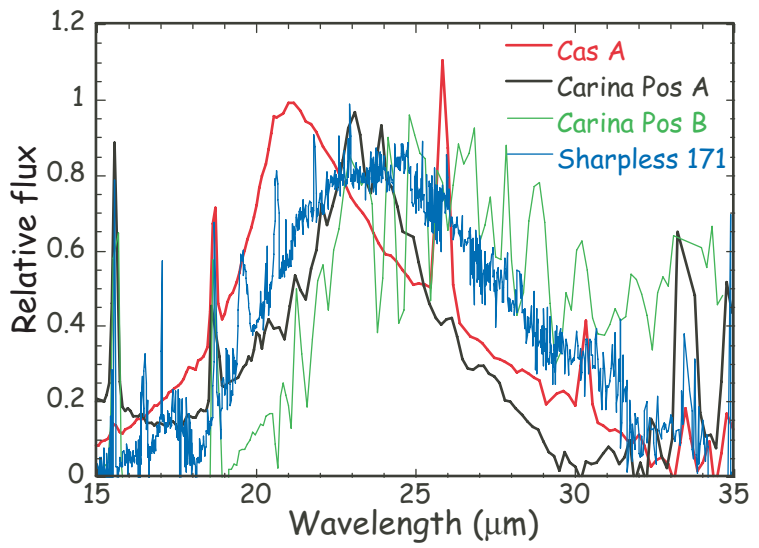

Figure 3. Comparison of features around 22 $\mu \mathrm{m}$ in the various objects. The red line indicates the Cas A feature, the black line at a position of the Carina nebula, the green another position of the Carina nebula, and the blue line the feature in the Sharpless 171 spectrum. See Onaka et al. (2009) for details.

thus quite important to understand the dust formation process and to derive the physical conditions in ejecta.

As in observations of SNe, spectroscopy in a wide spectral range from MIR to FIR is crucial for observations of SNRs because of the multi-component nature and the presence of line emission and dust features in SNR spectra. Whereas FIR observations are indispensable to search for the cold dust component, high spatial resolution is needed to avoid confusion of overlapping emission in the line-ofsight and extract the IR emission from the ejecta. We propose to make MIR to FIR imaging spectroscopic observations of about 50 SNRs that will have been known to show IR emission with SPICA to study the nature of dust formed in SN ejecta. Analysis of the spectral energy distribution of the IR emission in MIR to FIR allows us to study the interaction with the surroundings and the dust processing in SNe (Seok et al., 2008). High spectral resolution observations of the $\mathrm{CO}$ fundamental in $4.5-4.7 \mu \mathrm{m}$ will also be interesting.

\section{Dust SUPPly FROM LOW-MASS EVOLVED STARS}

Mass-loss process in AGB stars and PNe has been studied extensively in the IR. However the initiation of the massloss flow near the photosphere and the relation between the dust formation and the pulsation are not yet fully understood (e.g. Höfner, 2009). FIR observations of dust shell around low-mass evolved stars show that the massloss is not steady as usually assumed, but is rather sporadic (e.g. Izumiura et al., 1997, 2009, references therein). FIR observations of finer spatial resolution will certainly enable much more detailed investigations on the history of mass-loss, leading to an accurate estimate of the dust supply rate from low-mass evolved stars.

Detection of carbon stars with oxygen-rich dust shells indicates the transition from oxygen-rich to carbon-rich atmosphere in AGB stars (Willems \& de Jong, 1986). High spatial resolution MIR spectroscopy of a planetary nebula with the Subaru telescope in fact reveals the presence of a silicate dust shell right outside the carbon-rich shell (Matsumoto et al., 2008a). Ground-based telescope has poor sensitivity for the diffuse emission in the MIR, which prevents us from making a further detailed study. SPICA will extend the study, making a significant step to the understanding of mass-loss process during the transition in chemistry of the atmosphere owing to its high sensitivity to the diffuse emission and its good spatial resolution comparable to large ground-based telescopes in the MIR.

To understand mass-loss and dust formation in lowmass evolved stars, we propose to make MIR to FIR high spatial resolution imaging spectroscopy of about 30 AGB stars and planetary nebulae with SPICA. This study also allows us to investigate the possible formation of polycyclic aromatic hydrocarbons (PAHs), which are thought to be responsible for the unidentified infrared (UIR) bands ubiquitously seen in the ISM (e.g. Onaka et al., 1996), in carbon stars. High spectral resolution observations in MIR are important to significantly advance our understanding of "MOLsphere" detected in AGB stars by ISO observations (Tsuji, 2000) and its relation to mass-loss process.

\section{Material Circulation in our Galaxy}

To obtain a correct view of the distribution of dust-supplying stellar sources in our Galaxy we propose to make a MIR Galactic plane survey with SPICA. The proposed survey consists of imaging and spectroscopic observations. Spitzer GLIMPSE survey (Benjamin et al., 2003) has a detection limit of $0.5 \mathrm{mJy}$ at $4.5 \mu \mathrm{m}$ (Robitaille et al., 2007). It is also confused with sources in the Galactic plane. The flux of red clump stars at the other edge of the Galactic disk is estimate to be about $70 \mu \mathrm{Jy}$ at $5 \mu \mathrm{m}$, including the interstellar extinction. The source density down to this flux level is extrapolated from GLIMPSE data (Benjamin et al., 2005) to be 0.2 source in one $\operatorname{arcsec}^{2}$. Therefore SPICA survey will not be limited by confusion. Detection of a $70 \mu \mathrm{Jy}$ source at $5 \sigma$ can be reached in a net integration time of $3 \mathrm{~s}$ with MIRACLE. Ergo, the imaging survey to detect all red clump stars in our Galaxy can be completed in a relatively short time if an efficient survey capability of SPICA is equipped. For the imaging survey we propose to cover the region of $-90^{\circ}<l<+90^{\circ}$ and $-2^{\circ}<b<+2^{\circ}$. We also propose to make about 10 repeated observations to investigate the time variability. The imaging survey will provide us with the structure of our entire Galaxy, including the bar, bulge, and arms and the distribution of dust-supplying sources in our Galaxy. We expect to detect one billion sources in this survey.

The spectroscopic survey makes use of the multi-slit of MIRACLE and obtains spectra in the region of $-60^{\circ}<$ $l<+60^{\circ}$ and $-1^{\circ}<b<+1^{\circ}$. With the net integration time of $3 \mathrm{~s}$ per slit position, we will reach $600 \mu \mathrm{Jy}(5 \sigma)$ 
at $5 \mu \mathrm{m}$. Again we need an efficient slit scan capability with MIRACLE on SPICA. The spectroscopic survey is expected to detect one million sources, including very luminous red objects, which allows us to obtain the complete distribution of mass-losing massive stars in our Galaxy for the first time. It will also provide a complete diffuse emission map of the Galactic plane. Combining the results of this survey with the detailed study of SNe, SNRs, and low-mass evolved stars, we will be able to understand the contribution of each stellar source to the interstellar dust at every position of the inner part of our Galaxy.

\section{Material Circulation in Nearby Galaxies}

The study of the material circulation based on the dust cycle can be extended to the investigation on the material evolution in external galaxies. Observations of external galaxies further allow us to study the plasma interaction with dust grains, whose study is hampered severely by emission from the Galactic plane in observations of our Galaxy. In fact observations of elliptical galaxies associated with intense X-ray emission indicate different appearance of the UIR bands, in which the usually strong 6.2 and $7.7 \mu \mathrm{m}$ band emissions become weak or absent compared to the $11.3 \mu \mathrm{m}$ band emission (Kaneda et al., 2008). Similar variations in the PAH bands have also been reported in the interarm region of NGC6946 (Sakon et al., 2007) and the halo of M82 (Galliano et al., 2008), suggesting that the variations can be attributed to the plasma processing of the band carriers.

In this regard, the properties of dust grains in the halo region need to be explored in detail. Figure 4 shows the $7 \mu \mathrm{m}$ image (green) of M82 taken by $A K A R I /$ IRC superposed by the $\mathrm{H} \alpha$ emission image (red) from the SINGS dataset (Onaka \& Salama, 2009). It clearly shows that the $7 \mu \mathrm{m}$ emission is distributed over the $\mathrm{H} \alpha$ emission up to several kpc from the galactic disk. The AKARI $7 \mu \mathrm{m}$ image is supposed to be dominated by the UIR band emission. In fact the $17 \mu \mathrm{m}$ UIR band complex is detected in the extended emission by Spitzer/IRS (Engelbracht et al., 2006). Since the UIR band carriers should be destroyed efficiently in harsh environments, how they are brought and survive there is an important issue for the understanding of the dust life cycle in galaxies. The presence of the UIR band emission in the outflow from the galactic disk has also been detected in another dwarf galaxy NGC1569 by $A K A R I$ observations, where the band carriers are likely to be produced in shocks by the fragmentation of large carbonaceous grains (Matsumoto et al., 2008b).

Survival of the UIR band carriers should be investigated relative to the abundance of sub-micron dust grains to scrutinize the variation in the dust size distribution. Hence, FIR observations of extended structures of galaxies with similar spectral resolution are indispensable. Figure 5 shows the AKARI $90 \mu \mathrm{m}$ image with the ROSAT/HRI Xray contours of NGC253 (Kaneda et al., 2009). It indicates

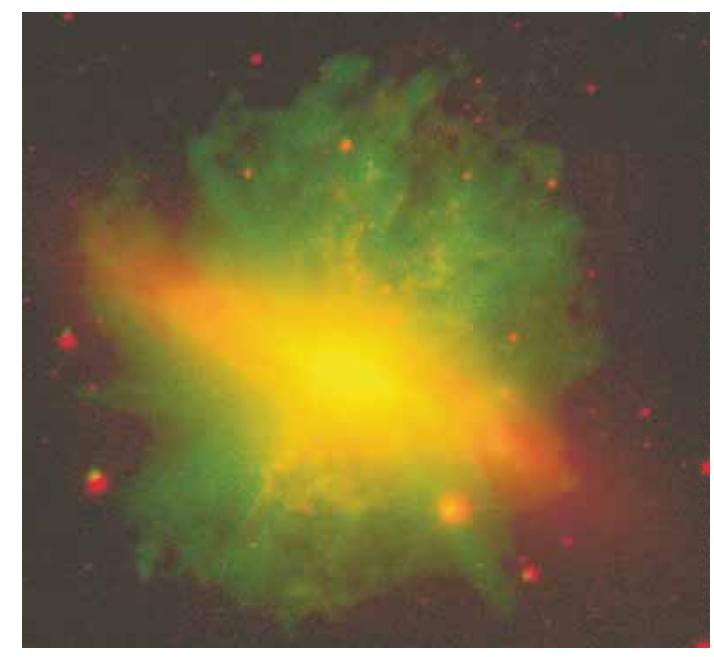

Figure 4. AKARI 7 4 m image of M82 (green) superposed with

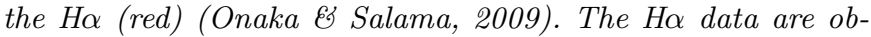
tained from the SINGS project (Kennicutt et al., 2003). The image size is $10^{\prime} \times 10^{\prime}$.

FIR excess emission at both ends of the upper galactic halo, which is associated with X-ray emission. Dust grains that emit FIR excess radiation must have been entrained by the galactic outflow and survive for several tens Myr at most (Kaneda et al., 2009). The spectral energy distribution from MIR to FIR provides us with crucial information on the variation in the dust size distribution in extended structures. Similar studies for the extended structure seen in the MIR of M82 and NGC1569, however, require much higher spatial resolution and better sensitivity in the FIR.

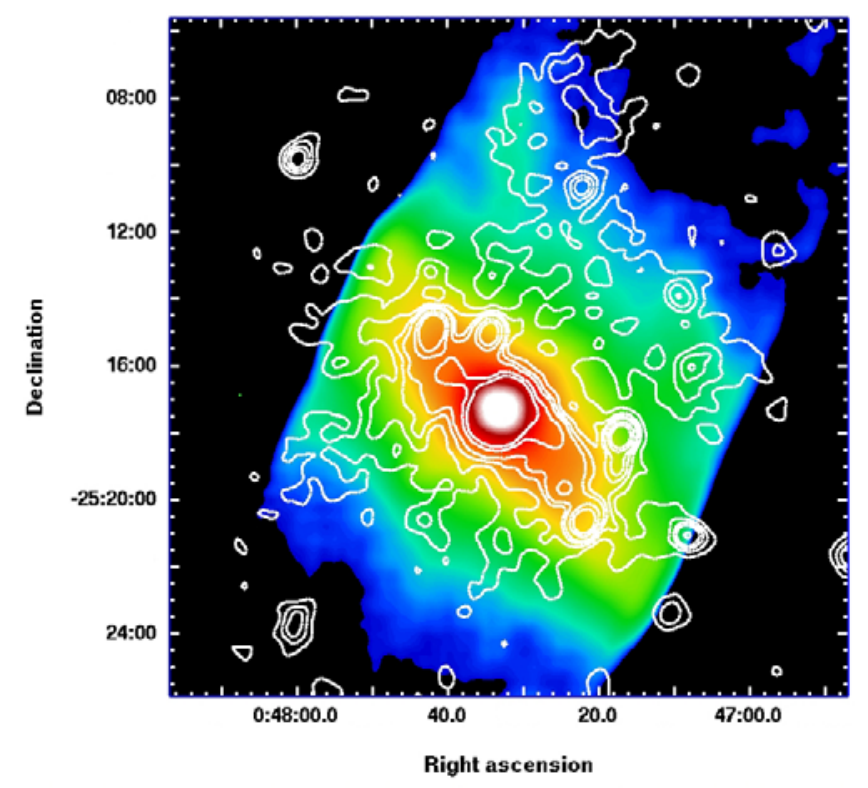

Figure 5. AKARI $90 \mu \mathrm{m}$ image in color superposed with the ROSAT/HRI contours (Kaneda et al., 2009). 
Halos of external galaxies provide unique physical conditions, where dust destruction process in the plasma environment can be investigated intensively with MIR and FIR observations, while observations nearly face-on galaxies enable us to study the role of individual objects, such as, HII regions, molecular clouds, and galaxy nuclei, in the dust life cycle in the universe without being confused by other sources. We propose to make imaging spectroscopic observations of 50-100 nearby galaxies in the MIR to FIR with SPICA and study the dust life cycle in the universe, including the feedback from nuclear activities, the effect of merging on material evolution, the origin and evolution of carbonaceous and silicate grains. High spatial resolution imaging spectroscopy in the MIR and FIR is also useful to study emission lines of gas species from the various regions in galaxies (e.g. Negishi et al., 2001).

\section{DUST FORMATION IN DENSE INTERSTELLAR CLOUDS}

Dust formation in dense clouds has not been studied in detail yet. A laboratory study suggests a possibility of silicate formation in cold environments (Nuth \& Moore, 1988), but it is still quite uncertain what kind of dust will indeed grow in dense clouds. Here we discuss a model of dust species that could be formed in dense ISM and emphasize the importance of spectroscopy in $20-50 \mu \mathrm{m}$ for the study of the ISM dust.

Bradley (1994) and Bradley et al. (1999) suggest an interesting possibility that "glass with embedded metal sufides" (GEMS) grains found in interplanetary dust particles (IDPs) are a good ISM dust analog. GEMS contains sulfur, which is not depleted in the diffuse ISM (Savage \& Sembach, 1996). However sulfur is largely depleted in dense clouds (Joseph et al., 1986), suggesting that dust formed in dense regions may contain sulfur as GEMS. Keller et al. (2002) indicate that GEMS in IDPs have characteristic band features in the $20-30 \mu \mathrm{m}$ region.

Figure 6 shows the absorption efficiency of $\mathrm{MgS}$ and FeS grains with a spherical shape and a shape distribution. MgS grains have a broad feature around $30 \mu \mathrm{m}$, whereas FeS grains show several band features in $20-50 \mu \mathrm{m}$. Broad features around $30 \mu \mathrm{m}$ have been observed in a number of carbon stars, which have been successfully accounted for by MgS grains (Hony et al., 2002). To our knowledge a similar broad $30 \mu \mathrm{m}$ feature in the ISM has so far been detected only towards the Galactic center (Chan et al., 1997). The spectral region in $20-50 \mu \mathrm{m}$ has not been explored with sensitive instruments and contains a number of interesting solid features untouched. We propose to make high-sensitivity spectroscopy in MIR to FIR of dense cloud regions to investigate the dust species formed there with the SPICA instruments.

High sensitivity spectroscopy with medium spectral resolution also enables us to detect a number of emission lines from ions, atoms, and molecules in dense regions. A recent study of Spitzer observations indicates a large abun-

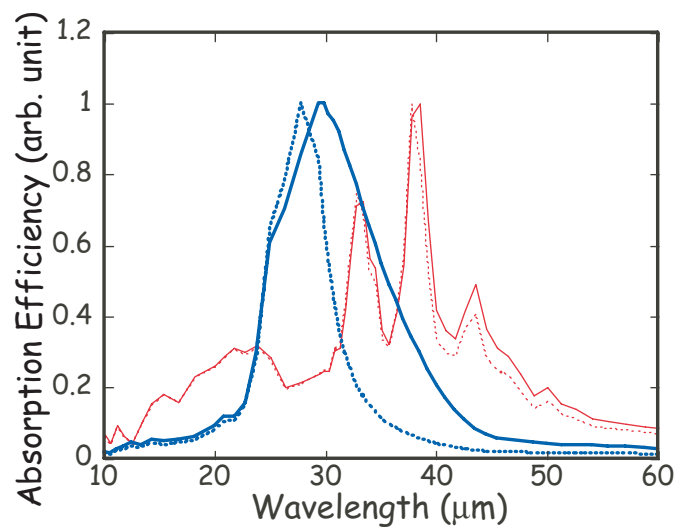

Figure 6. Absorption efficiency of $M g S$ (thick lines) and $\mathrm{FeS}$ (thin lines) grains normalized at their peak. The solid lines indicate those of the continuous distribution of ellipsoids (CDE) with the weight on spherical shape (Ossenkopf et al., 1992), while the dotted lines show those of spherical grains. The optical data are taken from Begemann et al. (1994).

dance of silicon in the gas phase, suggesting the presence of Si-bearing dust species other than silicates (Okada et al., 2008) and the importance of IR gas spectroscopy in dense star-forming regions (Okada et al., 2009). The proposed study will give us a complete view of dust and gas chemistry in the ISM that has not been unveiled and open up a new research field in astronomy.

\section{Summary}

The dust life cycle in the ISM is a key process for the material evolution in galaxies and the universe. The study of the dust and gas properties in the various ISM environments requires sensitive and high spatial resolution observations in $10-200 \mu \mathrm{m}$, where a number of important solid features as well as emission lines from gas species reside. For this study, the medium resolution spectroscopy $(R \sim 1000)$ and imaging of a wide field-of-view $(\sim \mathrm{a}$ few arcminute) are required to investigate extended objects and obtain the spectral energy distribution and line and band features. High spatial resolution, particularly in the FIR, is indispensable. Efficient survey capability is required for the MIRACLE Galactic plane survey. Higher resolution spectroscopy in the MIR adds unique information on this study. SPICA provides the facility with the unprecedented sensitivity in this spectral range. The proposed study will unveil the origin, processing, and death of dust grains in the universe, providing us with a completely new view of interstellar dust grains.

\section{ACKNowledgements}

This presentation was prepared on the basis of the discussions with the SPICA science working group of the interstellar medium in Japan. The authors are grateful for the contributions from all the Japanese ISM science working group members. This work uses the data based on observations of $A K A R I$, a JAXA project with the participation of ESA and 
those taken by Spitzer Space Telescope, which is operated by the Jet Propulsion Laboratory, California Institute of Technology under a contract with NASA. This work is in part supported by a Grant-in-Aid from the JSPS (18204014).

\section{REFERENCES}

Benjamin, R. A., Churchwell, E., Babler, B. L., et al. 2003, PASP, 115, 953

Benjamin, R. A., Churchwell, E., Babler, B. L., et al. 2005, ApJL, 630, L149

Bradley, J. P. 1994, Science, 265, 925

Bradley, J. P., Keller, L. P., Snow, T. P., et al. 1999, Science, 285, 1716

Begemann, B., Dorschner, J., Henning, Th., Mutschke, H., \& Thamm, E. 1994, ApJL, 423, L71

Chan, K.-W., Moseley, S. H., Casey, S., et al. 1997, ApJ, 483,798

Chan, K.-W., \& Onaka, T. 2000, ApJL, 533, L33

Clayton. D. D., Liu, W., \& Dalgarno, A. 1999 Science, 283,1290

Clayton, D. D., Deneault, E. A.-N., \& Meyer, B. S. 2001, ApJ, 562, 480

Engelbracht, C. W., Kundurthy, P., Gordon, K. D., et al. 2006, ApJL, 642, L127

Galliano, F., Madden, S., Tielens, A. G. G. M., Peeters, E., \& Jones, A. P. 2008, ApJ, 679310

Ghavamian, P., Raymond, J. C., Blair, W. P., et al. 2009, ApJ, 696, 130

Höfner, S., 2009, in Proc. of Comic Dust - Near and Far, ASP Conf. ser., in press (arXiv:0903.5280)

Hony, S., Bouwman, J., Keller, L. P., \& Waters, L. B. F. M. 2002, A\&A, 390, 533

Izumiura, H., Waters, L. B. F. M., de Jong, T., et al. 1997, A\&A, 323, 449

Izumiura, H., Ueta, T., Yamamura, I., et al. 2009, in Proc. of AKARI a light to illuminate the misty Universe, ASP Conf. ser., in press

Jones, A. P., Tielens, A. G. G. M., \& Hollenbach, D. K. 1996, ApJ, 469, 740

Joseph, C. L., Snow, T. P., Jr., Seab, C., \& Crutcher, R. M. 1986, ApJ, 309, 771

Kaneda, H., Onaka, T., Sakon, I., et al. 2008, ApJ, 684, 270

Kaneda, H., Yamagishi, M., Suzuki, T. \& Onaka, T. 2009, ApJ, 698, L125

Keller, L. P., Hony, S., Bradley, J. P., et al. 2002, Nature, 417,418

Kennicutt, R. C., Jr, Armus, L., Bendo, G., et al. 2003, PASP, 115, 928

Kotak, R., Meikle, P., Pozzo, M., et al. 2006, ApJL, 651, L117

Kotak, R., Meikle, W. P. S., Farrah, D., et al. 2009, ApJ, submitted (arXiv:0904.3737)

Lee, H.-G., Koo, B.-C., Moon, D.-S., et al. 2009, ApJ, submitted
Matsumoto, H., Sakon, I., Onaka, T., et al. 2008a, ApJ, 677,1120

Matsumoto, H., Onaka, T., Sakon, I., \& Kaneda, H. 2008b, Proc. of IAU Symp. 251, 249

Nakagawa, T., \& Murakami, H. 2007, Adv. Sp. Res., 40 679

Negishi, T., Onaka, T., Chan, K-W., \& Roellig, T. L. 2001, A\&A, 375, 566

Nozawa, T. Kozasa, T., Umeda, H., Maeda, K., \& Nomoto, K. 2003, ApJ, 598, 785

Nuth, J. A., \& Moore, M. H. 1988, ApJL, 329, L113

Okada, Y., Onaka, T., Miyata, T., et al. 2008, ApJ, 682, 416

Okada, Y., Onaka, T., Kaneda, H., \& Sakon, I. 2009, this volume

Onaka, T. 2000, ISAS Report, SP14, 83

Onaka, T., Yamamura, I., Tanabé, T., Roellig, T. L., \& Lunming, Y. 1996, PASJ, 48, L65

Onaka, T., Matsumoto, H., Sakon, I., \& Kaneda, H. 2008, Proc. of IAU Symp. 251, 229

Onaka, T., \& Salama, A. 2009, Exper. Astron., in press

Onaka, T., Roellig, T. L., Okada, Y., \& Chan, K.-W. 2009, The Evolving ISM in the Milky Way and Nearby Galaxies, http://ssc.spitzer.caltech.edu/mtgs/ismevol/

Ossenkopf, V., Henning, Th., \& Mathis, J. S. 1992, A\&A, 261, 567

Rho, J., Kozasa, T., Reach, W. T., et al. 2008, ApJ, 673, 271

Rho, J., Jarret, T. H., Reach, W. T., et al. 2009a, ApJL, 693, L39

Rho, J., Reach, W. T., Tappe, A., et al. 2009b, ApJ, 700, 579

Robitaille, T. P., Cohen, M., Barbara, W. A., et al. 2007, AJ, 134, 2099

Roche, P., Aitken, D., \& Smith, C. 1991, MNRAS 252, $39 \mathrm{P}$

Sakon, I., Onaka, T., Wada, T., et al. 2007, PASJ, 59, S483

Sakon, I., Wada, T., Ohyama, Y., et al. 2009, ApJ, 692, 546

Savage, B. D., \& Sembach, K. R. 1996, ARA\&A, 34, 279

Seab, C. G. 1988, in Dust in the Universe, (Cambridge Univ. Press, Cambridge), 303

Seok, J.-Y., Koo, B.-C., Onaka, T., et al. 2008, PASJ, 60, S453

Swinyard, B., Nakagawa, T., Merken, P., et al. 2009, Exper. Astron, 23, 193

Tappe, A., Rho, J., \& Reach, W. T. 2006, ApJ, 653, 267

Tielens, A. G. G. M. 2005, The Physics and Chemistry of the Interstellar Medium, Cambridge University Press

Todini, P., \& Ferrara, A. 2001, MNRAS, 325, 726

Tsuji, T. 2000, ApJL, 540, L99

Willems, F. J., \& de Jong, T. 1986, ApJL, 309, L39

Wooden, D. H., Rank, D. M., Bregman, J. D., et al. 1993, ApJS, 88, 477 